\title{
MODELLING AND SIMULATION FOR CONTROL OF A MEMS ELECTROMAGNETIC SCANNER
}

\author{
Comeaga Constantin Daniel ${ }^{1}$, Morega Alexandru Mihail ${ }^{2}$, Gheorghe Viorel ${ }^{1}$, Ilie Cristinel ${ }^{3}$ \\ ${ }^{1}$ Mechatronics and Precision Engineering Department, Politehnica University of Bucharest, \\ Splaiul Independenţei, nr. 313, Bucharest, Romania, \\ ${ }^{2}$ Machines, Materials and Electric Devices Department, University POLITEHNICA of Bucharest, \\ 313 Splaiul Independentei st., Bucharest, Romania, \\ ${ }^{3}$ National Institute for Research and Development in Electrical Engineering ICPE-CA, \\ Splaiul Unirii, Nr. 313, District 3, 030138, Bucharest, Romania \\ E-mails: comeaga daniel@yahoo.com, amm@iem.pub.ro, viorel gheorghe@yahoo.com, cristinel.ilie@icep ca.ro
}

\begin{abstract}
The article presents the mathematical modelling and simulation models of a MEMS electromagnetic scanning system, with the aim to discuss sources of nonlinear behavior and to compare voltage vs. current control modes. The electromagnetic (EM) actuators develop high forces at any scale, and can be used at both resonance or far from it. This study completes previous design and manufacturing work of the electro-mechanical structure and its electric supply circuits. A nonlinear mathematical model for a general case, with the main actuator connected to a second elastic system carrying the mirror, and the particular mathematical model for infinite contact impedance between the actuator and the mirror support, allowing use of a single degree of freedom model for the mechanical part, are presented. The models are simplified for current control strategy discussion, and their SIMULINK implementation is included altogether with the results of simulations that confirm the advantages of the current control.
\end{abstract}

Keywords: MEMS, Electromagnetic Actuator, Electromagnetic Scanner, Nonlinear Model, Numerical Simulation

\section{Introduction}

LASER micro scanning devices that use electromagnetic actuators (EA) are widely used in a large scale of applications, from virtual reality devices to miniature medical equipment. EAs have complex structures but develop high forces at any scale $[1,2]$. A very important argument in using EA scanners is a low sensitivity to dimensional imperfections, allowing for laser LIGA technology usage. Few designs are available though, from low frequency devices working out of resonance (at driving frequencies maximum $10 \%$ of the first resonance frequency) to resonant-frequency devices. This paper continues previous work on new type of EAs [3] usable in micro-mirrors positioning devices that deflect the light beam about one or two axes, which are part of laser processing stations that may replace conventional scanners. The EA is provided with a fixed coil and a moving, permanent magnets array (PMA). The actuation is achieved through the interaction between the PMA and the electrical current.

\section{The Electromagnetic Actuator in MEMS}

Two designs are available for EAs, one with mobile coil and another with mobile magnets. The first design, commonly referred to as electro-dynamic, is based on the interaction between a fixed magnetic field and an electric current.

The second type, which is called electromagnetic at macro scale, is based on the property of magnetic circuits to reach a minimum potential magnetic energy state [4]. A simple solution is to use a mobile part made of a high magnetic permeability material placed in the magnetic field produced by an electrical current carrying coil (as in bulk electromagnets). This design is difficult to miniaturize because both the mobile and the fixed parts that make the magnetic circuit must to be fabricated of good magnetic materials. An alternative design uses magnets placed on a mobile element and fixed electrical current coils.

For rotational systems like scanners the main problem is to acquire equivalent torque acting on the magnetic material. As it is practically impossible to 
change the magnetization in the surface of magnets matrix at this scale (the permanent magnet(s) are deposited in one step and the magnetization is obtained either in this step or in an additional step, but is impossible to produce a strong and fast varying magnetization field at microscale) the single solution could be the usage of a special design for the coil. The simplest EA solution is based on an elastic beam [5-6] but proved to provide only low rotational angles of the mirror.

A possible solution is to change the magnetic field produced by the coils, at a micro-scale, for high values of magnetic flux density, but this is very difficult because it requests complicated coil designs. Another solution is to convert a displacement in rotation by using a simple mechanism, which could have an important advantage, considering that micro-scanners are usually integrated in mobile systems, tending to became autonomous systems by using integrated energy recovery. The translational system could be set easily into vibration by external means, allowing its utilization as an energyharvesting device. So, the combined translational rotational system with a mobile magnet could be used for bi-directional energy conversion. Thus, the energy conversion efficiency is good in both wise, either for mirror positioning or for harvesting energy also used in the scanner.

Previous work [3] focused on different types of magnetization (in EA plane and out-of-EA plane) proving the horizontal one (Fig. 1) is more stable, even though the forces are lower.

The magnets array was chosen because previous studies on bulk film magnet show defects and non-uniform magnetic parameters. The array structure removes these problems, even though a volume of the magnets is sacrificed for gaps, and the overall performances are improved.
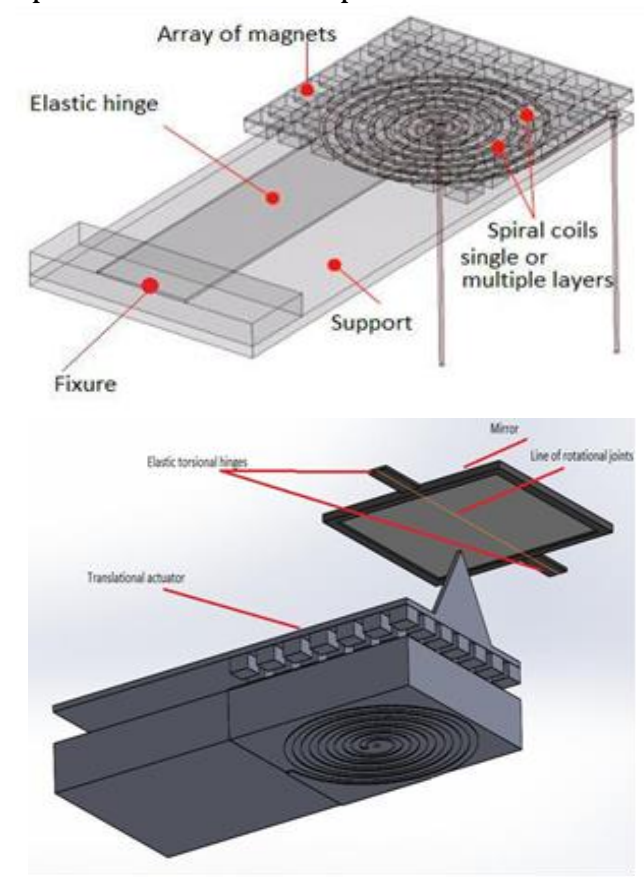

Figure 1: The CAD model of the EA and the CAD model of the EA with mechanism

An improvement of the bending beam design, aimed to compensate for the low rotations obtained even for large translations, may be a tangent mechanism (Fig. 1) used to drive the mirror. The contact point between the beam and the mirror is placed before the line linking the torsion elastic hinges of the mirror, thus assuring a large amplification.

\section{The Linear Mathematical Modelling}

The actuator modelling could be performed in two ways: either based on continuum medium or based on discrete/lumped parameters models.

The first technique was used to prove the feasibility of the actuator and for the preliminary design of the actuator and electric circuit $[3,5,6,7]$.

The lumped parameters model is necessary and allows for a global simulation, which is useful in the design of the control algorithm and the circuit. It may produce also the values of the lumped parameters that optimize the control.

The simplest model assumes a mechanical system with one degree-of-freedom (mass-stiffnessviscous damping, Fig. 1,a) and a series inductance resistance branch in parallel with a capacitance for high frequencies, Fig. 1,b)). The mechanical and electrical sub-systems are connected via a transformer with, for the simplest model, a linear relation electric current - mechanical force, respectively between the velocity and induced voltage.

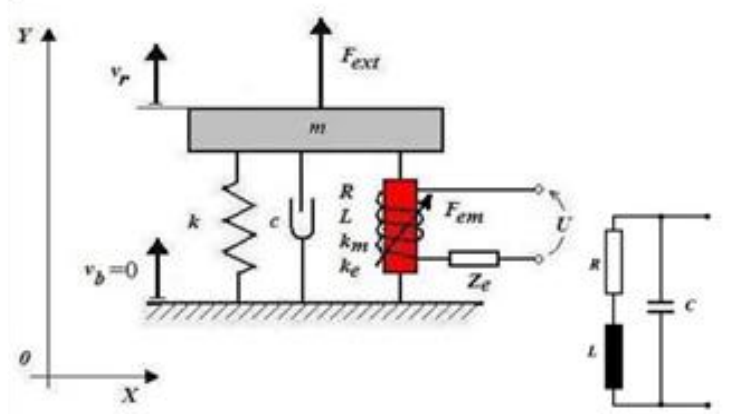

Figure 2: The 1 DOF electro-mechanical equivalent model: a) 1-DOF mechanical model; b) simplified electrical impedances $Z_{e}$ [7]

The simplest model used for simulation assumes linear behavior only, and it is indicated in (1) for voltage control.

$$
\left\{\begin{array}{l}
\left(m \cdot s+c+\frac{k}{s}\right) \cdot v_{r}(s)-k_{m} \cdot i(s)=F_{e x t}(s) \\
k_{e} \cdot v_{r}(s)+(R+L \cdot s) \cdot i(s)+Z_{e} \cdot i(s)=U(s)
\end{array}\right.
$$

where m- mobile mass; k- stiffness coefficient of the equivalent elastic component; c- damping 
coefficient; $\mathrm{k}_{\mathrm{m}}$ - coefficient linking electric current and electromagnetic force; $\mathrm{ke}^{-}$coefficient linking the velocity and induced voltage; R-resistance of the electric circuit; L- inductance of the electric circuit; $\mathrm{Z}_{\mathrm{e}}$ - external electric inductance.

An improved model assumes the nonlinearities arising from mechanical and electromagnetic physics:

- nonlinear behaviour of the elastic material (nonlinearity and hysteresis),

- nonlinear relation between the distributed force acting on the magnets and the displacement, due to large displacements,

- nonlinear relation between the displacement and magnetic force due to the magnetic flux drop when the gap between magnets and coil rise,

- nonlinear relation between the electric voltage and current due to a variation of inductance with the gap, - nonlinearity of the induced voltage w.r.t. the speed of magnets (the ke coefficient),

- nonlinear relation between the electric current and force due to the gap variation (the km coefficient).

The combined actuator with rotation and translation has a more complex structure. If the actuator uses a pure translation of the magnets array and it is provided with a pure mechanical connection to a rotation system, then the model may be improved by introducing in the first eq. (1) a supplementary force and by adding a supplementary equation for the mirror motion.

Usually, one of the mechanical systems is driven at resonance and the second is driven away of resonance, allowing the removal of the inertial and damping terms from the out-of-resonance system, and further recasting the system back into two equations.

A relation between rotation and translation may be easily obtained for a simple, single-beam system, which deforms due to the magnetic force applied on the magnets such as to produce a displacement and consequently a rotation of the magnetic array. So, even though the magnetic force depends on both translation and rotation, and the inductance variation alike, it can be assumed that only one mechanical variable occurs.

\section{The Nonlinear Mathematical Modelling}

A model closer to reality uses lookup tables to introduce the non-linearity between force and displacement, due to the mechanical part, between current and force, due to the magnetic flux density variation with magnets positions, between positions and inductance.

The model is more complex if all nonlinearities are considered. In the general case, with two mechanical variables (translation perpendicular to the magnets plane and rotation of the mirror with respect to an axis contained in the mirror plane - the line of rotational joints, Fig. 1) and one electrical variable (the coil current) the equations are nonlinear:

$$
\begin{gathered}
M \frac{\mathrm{d}^{2} w}{\mathrm{~d} t^{2}}=F_{\text {emag }}-C_{\text {trans }} \frac{\mathrm{d} w}{\mathrm{~d} t}-w \cdot K_{\text {trans }}(w)-C p l_{\text {trans }}(w, \theta), J \frac{\mathrm{d}^{2} \theta}{\mathrm{d} t^{2}}=M_{\text {emag }}-C_{\text {rot }} \frac{\mathrm{d} \theta}{\mathrm{d} t}-\theta \cdot K_{\text {rot }}(\theta)-C p l_{\text {trot }}(w, \theta) \\
\operatorname{Mechanism}\left(C_{\text {trans }}, C p l_{\text {rot }}, w, \theta\right)=0 \\
U=\frac{\mathrm{d} \Psi}{\mathrm{d} t}+R i+U_{w}+U_{\theta} ; \Psi=L(w, \theta, i) \cdot i .
\end{gathered}
$$

where

- the translational subsystem in described by mass, $\mathrm{M}$, stiffness, $K_{\text {trans, }}$ and damping coefficient $\mathrm{C}_{\text {trans, }}$

- the rotational subsystem is described by rotational inertia, J, stiffness, $\mathrm{K}_{\text {rot }}$ and damping coefficient, $\mathrm{C}_{\text {rot, }}$ and

- the electrical circuit is characterized by resistance, $\mathrm{R}$, inductance, $\mathrm{L}$, and magnetic flux, $\Psi$.

The mechanical coupling between the mechanical subsystems is descried by the force, $\mathrm{C}_{\text {pltrans, }}$ the torque, $\mathrm{C}_{\text {plrot, }}$ and additional equations (called Mechanism). The equations depend on the type of mechanism, linking force, torque, displacement, and rotation.
The electromagnetic field acts on the mechanical systems with a force $\mathrm{F}_{\text {emag }}$ and a torque Memag.

The motion-induce voltages due to the magnetic flux variations are $U_{w}$ due to translation, $w$, and $U_{\theta}$ due to the rotation, $\theta$.

The coefficients are nonlinear, depending on variables

$$
\begin{aligned}
& U_{w}=\text { function }\left(w, \theta, i, \frac{\mathrm{d} w}{\mathrm{~d} t}\right) \\
& U_{\theta}=\text { function }\left(w, \theta, i, \frac{\mathrm{d} \theta}{\mathrm{d} t}\right) \\
& F_{\text {emag }}=f\left(w, \theta, i, \frac{\mathrm{d} x}{\mathrm{~d} t}\right)
\end{aligned}
$$




$$
M_{\text {emag }}=f\left(w, \theta, i, \frac{\mathrm{d} x}{\mathrm{~d} t}\right)
$$

The nonlinearities are descried by dependencies of the coefficients to the variables:

- Stiffness depends on the associated deformation/displacement, which for large deformations yields $K_{\text {trans }}=$ function $(w)$, $K_{\text {rot }}=$ function $(\theta)$;

- Damping was assumed viscous (the damping force proportional with the speed), hypothesis valuable for movement in fluids, including air as in this case. In practice, internal damping within the material could occur, and it is nonlinear (usually, hysteresis). Magnetic damping could occur also, due to the motion of conductive materials w.r.t. a magnetic field, nonlinear too. These nonlinearities are modelled through $C_{\text {trans }}=$ function $(w, i)$, $C_{r o t}=$ function $(\theta, i)$. The magnetic damping could be neglected due to the low magnetic field produced by the coils currents, and the low speed of the scanning systems. Also, the material used for the elastic beam has a very low hysteresis. Here we assume a linear viscous damping, i.e., $\mathrm{C}_{\text {trans }}=$ constant, $\mathrm{C}_{\text {rot }}=$ constant .

$$
K_{e, t r a n s}(w, \theta, i) \cong K_{e, t r a n s}\left(w_{0}, \theta_{0}, i_{0}\right), K_{e, r o t}(w, \theta, i) \cong K_{e, r o t}\left(w_{0}, \theta_{0}, i_{0}\right)
$$

The electromagnetic force and torque occur because the system is to attain the state having the lowest magnetic energy. For electrodynamic actuators (either translational or rotational), force and torque are proportional to the inducing current by Lorentz forces acting on conducting wires placed in external magnetic fields because the magnetic flux density produced by magnets is perpendicular to that selfinduced magnetic field, due to the coil movement, so it is almost constant (the coil is never removed from the magnetic circuit).

In this case the magnetic field varies with the positions of the magnets from few reasons:

- The magnetic field produced by the planar coil varies with the direction of motion;
- Voltages induced by motion are assumed linear w.r.t. velocity for common electro-dynamic actuators (modelled through coefficients $\mathrm{K}_{\mathrm{e}}$, trans and $\mathrm{K}_{\mathrm{e}}$, rot). These assumptions are valid for actuators with moving coils in large and constant magnetic field, such as in linear and rotational electro-dynamic actuators. For this type of electromagnetic actuator with strong variations of the magnetic field on the movement direction, mainly for translation, it is possible to assume a linear dependency on the translational and rotational speeds only for small speeds values around an equilibrium,

In general, when the magnetic field depends on the magnets positions and the electric current, the coefficients are generally functions of positions and electric current:

$U_{w}=K_{e, t r a n s}(w, \theta, i) \cdot \frac{\mathrm{d} w}{\mathrm{~d} t}, U_{\theta}=K_{e, r o t}(w, \theta, i) \cdot \frac{\mathrm{d} \theta}{\mathrm{d} t}$

For low amplitude motion about the equilibrium the coefficients are constants. The next equation describes this case where subscript 0 indicate a static equilibrium position (current i0 is produced by a DC voltage, over which is applied a small AC signal for obtaining oscillations), i.e.,

$$
K_{m, \text { trans }}(w, \theta, i) \cong K_{m, \text { trans }}\left(w_{0}, \theta_{0}, i_{0}\right), K_{m, \text { rot }}(w, \theta, i) \cong K_{m, \text { rot }}\left(w_{0}, \theta_{0}, i_{0}\right)
$$

- The magnetic flux density could be assumed proportional to the electric current. The coefficient, an inductance, depends on the positions and electric current.
- The magnetic field depends on the inducing electric current, which in turn depend on the inductance (so of the magnets position) and external voltage, assumed constant.

For small electric current variations, it is possible to assume a linear dependence of force/torque on the current, with coefficients depending on positions and electric current, i.e., $F_{\text {emag }}=K_{\text {m,trans }}(w, \theta, i) \cdot i, M_{\text {emag }}=K_{m, r o t}(w, \theta, i) \cdot i$, (6)

This case is far from the real situation, for scanning tasks with voltage control, but it is useful for the steady state voltage control or for current control of the actuator.

Again, for low amplitude movement about the equilibrium the coefficients are constants, i.e.,

- The coupling between rotation and translation is nonlinear for tangent or similar mechanisms. 
A simpler model assumes that the coupling torque is directly proportional to the coupling force.

An additional equation, depending on movements, is needed to link the coupling force and the relative displacements in the contact point.

For a nonlinear coupling the Mechanism equation becomes:

$$
C p l_{\text {rot }}(w, \theta)=f(w, \theta) \cdot C p l_{\text {trans }}(w, \theta)
$$

$$
C p l_{\text {rot }}(w, \theta)=c t \cdot C p l_{\text {trans }}(w, \theta), C \operatorname{Cpl}_{\text {trans }}(w, \theta)=\operatorname{Impedance}(w, \theta) \cdot\left(w-\frac{1}{c t} \theta\right)
$$

If the contact between the translational and rotational systems is rigid then the impedance is infinite, so the Mechanism equations become:

$$
C p l_{\text {rot }}(w, \theta)=c t \cdot C p l_{\text {trans }}(w, \theta), \quad w=\frac{1}{c t} \theta
$$

In this last case, the three-variable system yields a two-variable system ( $\mathrm{w}$ and $\mathrm{i}$ ).

\section{The Simulink Models}

The hypotheses for simulation model are:

- nonlinear stiffness, due to large deformations,

- linear damping (negligible mechanical hysteresis because the load is limited so as the stress is under $70 \%$ of the elasticity limit and no magnetic damping because the speeds are low; the material for elastic beam is a CuBe alloy allowing a high deformation without attaining the elasticity limit),

- small speeds allowing use of (4-5) (the first resonance is around few hundred hertz), and

- small electric currents allowing (6-7) (the very thin electric circuit obtained on printed circuit boards limit drastically the current values).

Null initial pretension is assumed, i.e. $\mathrm{C}_{\text {plrot }}(0,0)=0, \mathrm{C}_{\text {pltran }} \mathrm{S}(0,0)=0$.

When applying Laplace transform to equations (2) with simplifications (3) and (5) and considering relations expressed by equations (4/6) it is possible, in the hypothesis of small variations of position parameters and low variations speeds (a frequency content of signals far under the first resonance frequencies) to consider that the Laplace equations are established for constant values of $K_{e}$ trans, $K_{e}$ rot, $K_{m}$ trans,$K_{m}$ rot and coupling constants from (8-9), as done for a pure linear system and then, during simulations steps, the values of $\mathrm{K}_{\mathrm{e}}$ trans, $\mathrm{K}_{\mathrm{e}}$ rot, $\mathrm{Km}$ trans, $\mathrm{K}_{\mathrm{m} \text { rot }}$ and coupling constants from eq. (10-11) are recalculated as functions of variables $\mathrm{w}, \theta$ and $\mathrm{I}$ at each step. This approach is commonly met because is easily to use in Simulink using Look-up functions.
Other possibilities are to approximate all function with linear functions around the equilibrium point (the parameters $K_{e}$ trans, $K_{e}$ rot, $K_{m}$ trans, $K_{m}$ rot and coupling constants from eq. (8-11) are computed for this equilibrium point) or to approximate the parameters $\mathrm{K}_{\mathrm{e}}$ trans, $\mathrm{K}_{\mathrm{e} \text { rot }}, \mathrm{K}_{\mathrm{m} \text { trans }}, \mathrm{K}_{\mathrm{m}}$ rot and coupling constants with polynomial functions so as, after replacing them in Eq. (2) to obtain equations with different powers of variables, easily to use with Laplace transform.

Preliminary experimental tests on the studied type of electromagnetic systems shown variations of parameters difficult to express as polynomials functions in respect to variables. The linearization around of equilibrium point is also difficult due to large variations of parameters.

The look-up tables seem to be the easier way because the variations can be obtained experimentally or using FEM simulations, so are the chosen way for simulation.

For simplifying the model, the coupling between translational and rotational parts was approximated using first order Taylor series of coupling functions because this coupling part is usually an elastic beam or similar, with small variation of strains to avoid permanent deformations.

The equations used for general simulation become:

$$
\begin{aligned}
& w=\frac{v}{s}, \quad \theta=\frac{\omega}{s} \\
& v=\frac{1}{s}\left[\begin{array}{c}
-K_{\text {trans }}(w) \cdot w-C_{\text {trans }} \cdot v-\frac{\partial C p l_{\text {trans }}}{\partial w} \cdot w- \\
-\frac{\partial C p l_{\text {trans }}}{\partial \theta} \cdot \theta+K_{m, \text { trans }}(w, \theta, i) \cdot i
\end{array}\right] \\
& \omega=\frac{1}{s}\left[\begin{array}{c}
-K_{\text {rot }}(\theta) \cdot \theta-C_{\text {rot }} \cdot \omega-\frac{\partial C p l_{\text {rot }}}{\partial w} \cdot w- \\
-\frac{\partial C p l_{\text {rot }}}{\partial \theta} \cdot \theta+K_{m, r o t}(w, \theta, i) \cdot i
\end{array}\right]
\end{aligned}
$$




$$
i=\frac{1}{s \cdot\left(L+i \frac{\partial L}{\partial i}\right)}\left[\begin{array}{c}
U-R \cdot i-K_{e, t r a n s}(w, \theta, i) \cdot v- \\
-K_{e, r o t}(w, \theta, i) \cdot \omega-i \cdot\left(\frac{\partial L}{\partial w} v+\frac{\partial L}{\partial \theta} \omega\right)
\end{array}\right]
$$

For rigid coupling between the two mechanical systems or the mirror placed on the beam the equations become

$$
\begin{gathered}
w=\frac{v}{s} \\
v=\frac{1}{s}\left[-\overline{K_{\text {trans }}}(w) \cdot w-\overline{C_{\text {trans }}} \cdot v+K_{\text {m,trans }}(w, \theta, i)\right]
\end{gathered}
$$

$i=\frac{1}{s \cdot\left(L+i \frac{\partial L}{\partial i}\right)}\left[\begin{array}{c}U-R \cdot i-K_{e, t r a n s}(w, \theta, i) \cdot v- \\ -i \cdot v \cdot \frac{\partial L}{\partial w}\end{array}\right]$ where $\overline{K_{\text {trans }}}(w), \overline{C_{\text {trans }}}$ correspond to the equivalent parameters after reducing the rotational system to the translational one.

The mathematical model simplifies for current control, reducing to the first two mechanical equation. The problem due to the nonlinear relation between current and magnetic force remain.

Previously was proposed an improved voltagecontrolled Howland current source, called Advanced Current Source [7].

The Simulink model for the voltage control and the previous hypothesis, split in blocks interfaced in the main application, is show in Figures 3-6.

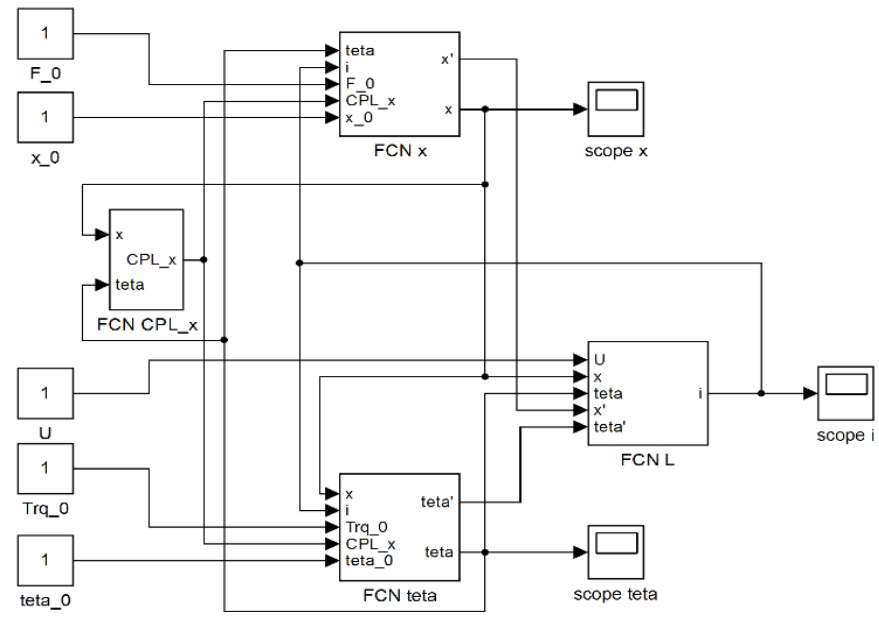

Figure 3: The global SIMULINK model
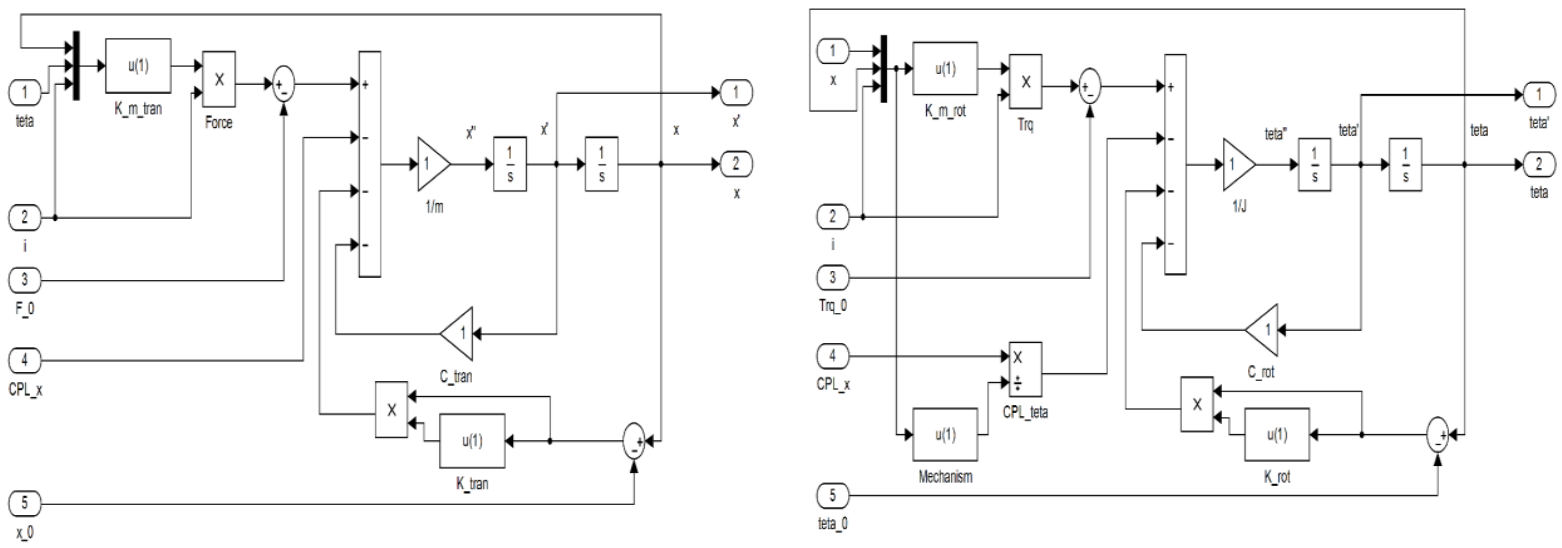

Figure 4: The SIMULINK model of the rotational subsystem and the model of the translational subsystem 


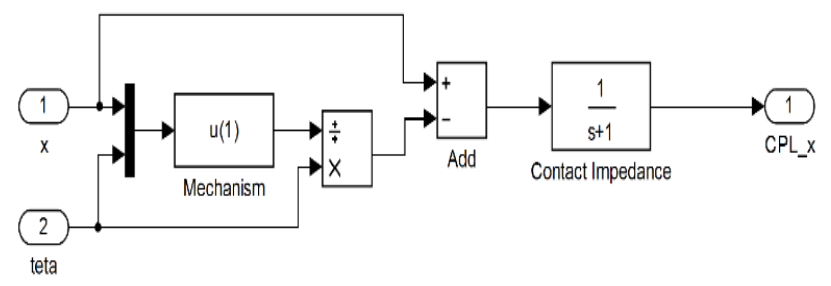

Figure 5: The SIMULINK model of the coupling subsystem

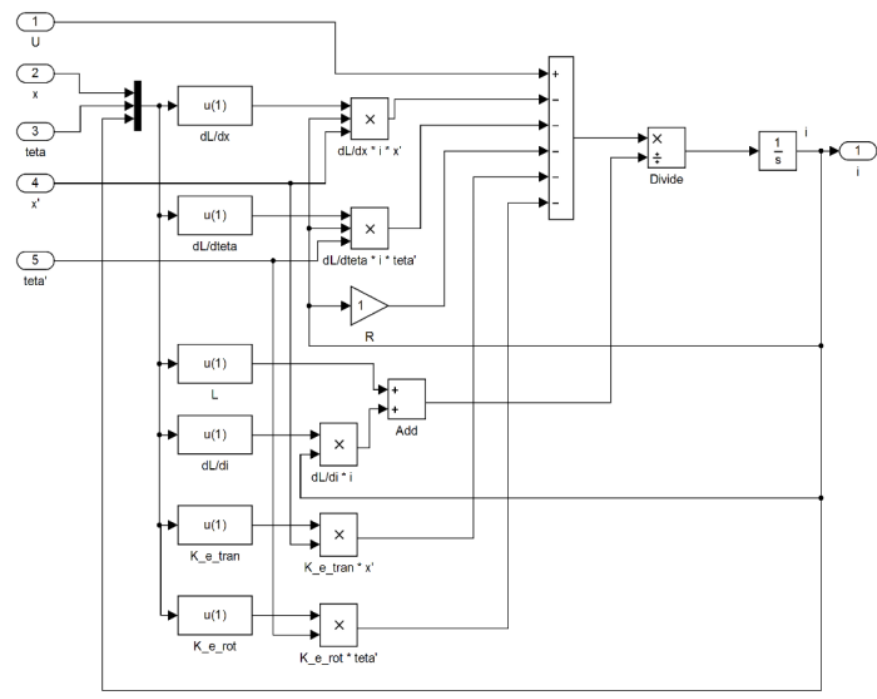

Figure 6: The SIMULINK model of the electric subsystem

The model parameters were derived through simulation using finite element methods $[3,5,6]$. The model assumes also a non-zero initial state, described by initial positions and forces at equilibrium. The simulation results for voltage and current control are in presented in Fig. 7.

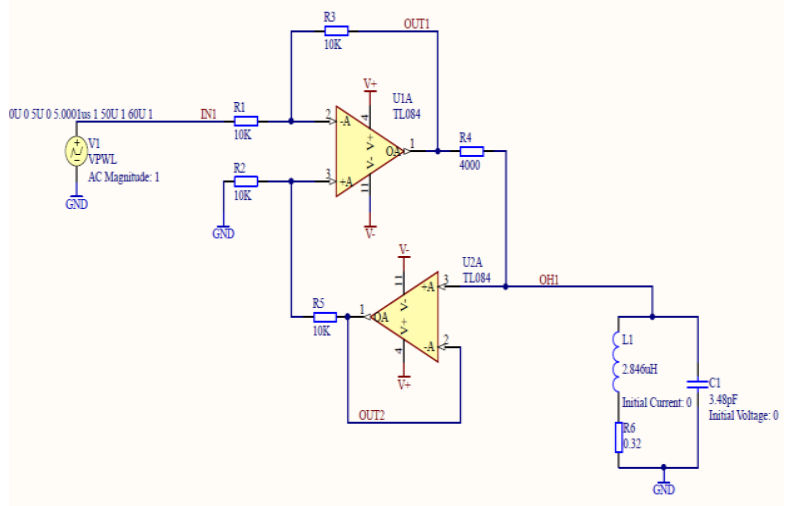

The voltage step is $22 \mathrm{mV}$. The current variation is a ramp with $2 \mathrm{~ms}$ rising time (selected based on frequency response of the driving circuit) and $33 \mathrm{~mA}$ stabilization value. The circuit used for current control is presented in Fig. 7 [8].

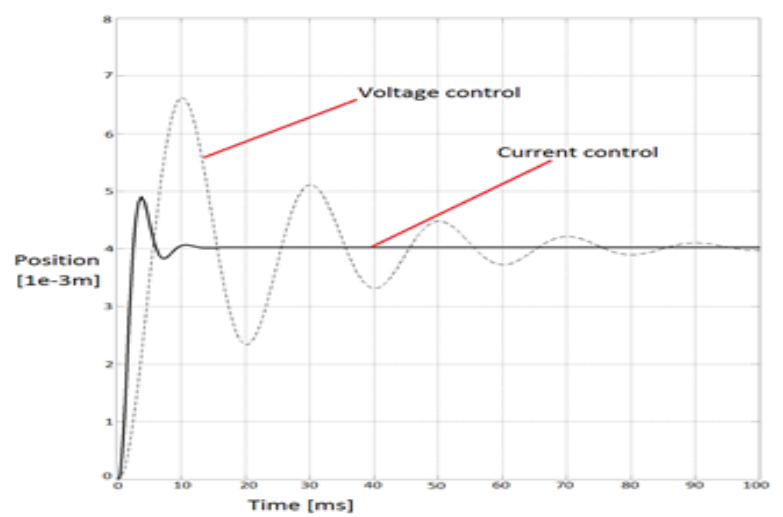

Figure 7: The electrical control circuit [7] and the control results

The simulations indicate the current control to be optimal, with oscillations reduced to $40 \%$ and a very low stabilization time (around 10ms for current control comparing to $100 \mathrm{~ms}$ for voltage control). The stabilization time is very important because is a factor limiting the scanning frequency. For voltage control the frequency is limited to $10 \mathrm{~Hz}$ while the current control allows a scanning frequency up $100 \mathrm{~Hz}$, enough for optical systems as micro- projectors (the minimum scanning frequency, due to the human eye response, is around $35 \mathrm{~Hz}$ but is recommended around $60 \mathrm{~Hz}$ ). It also provides for better compensation of nonlinearities.

Driving in current has few advantages, the most important being the removal of self-induced voltage influence at high velocities and also the reduce of inductance and resistance variations influence. 
The system became more robust to electromagnetic interference (appearing as a voltage perturbation) and to the parasitic movement of system base. For voltage control, the parasitic base movement is producing a supplementary electromagnetic selfinduced voltage superimposed on the control voltage, acting as a perturbation. Using current control, the base-movement induced voltage is removed and the system is more stable.

The model will be used for studies regarding the control strategies with the focus on nonlinearities impact removal.

As the intention is to derive a model useful for both situations (one or two masses) it is considered the most complex case with three variables - one electrical (voltage or current) and two mechanical ones (displacement of the array magnets and the mirror rotation).

\section{Conclusion}

A new type of electromagnetic scanning device was proposed based on the linear movement of an actuator using the interaction between a matrix of permanent magnets and a fixed coil. The linear actuator could drive a rotational mechanism, through an elastic link. The linear models currently used for electrodynamic actuators, as voice-coil actuators, have very limited applicability due to the strong nonlinearities in the new design.

A presentation of mechanical and electromagnetic sources of non-linearity is included. A nonlinear model was derived for the general case, with 2 degrees of freedom. It accounts for nonlinearities of the mechanical subsystems, a contact impedance between them, and also nonlinear behavior of the components in the electromagnetic circuits. The SIMULINK model is included and used for numerical simulation. The simplified mathematical model, with 1 degree-of-freedom, assuming perfectly stiff contact impedance between rotational and translational component, useful also if the mirror is fixed directly on the bending actuator, was derived together with the simulation model. All the models assume open loop control. The model of system with 1 degree-of-freedom is used for simulations of voltage and current control. The results confirmed the usefulness of current control for this type of actuator and also create a basis for further studies regarding stability and a possible feedback.

\section{Acknowledgements}

The work was conducted in the framework of AEEL PNCD II PT PCCA 249/2014 research grant, code PNII-PT-PCCA-2013-4-1557, financed by the Romanian National Authority for Research, Development and Innovation - UEFISCDI.

\section{References}

[1] Yan Jun, Luanava Selso and Casasanta Vincenzo, "Magnetic actuation for MEMS scanners for retinal scanning displays," in Proc. SPIE, vol. 4985, pp. 115-120, 2003.

[2] Laure K. Lagorce, Oliver Brand and Mark G, Allen, "Magnetic microactuators based on polymer magnets", IEEE J. Microelectromech. Syst., vol. 8 (1), pp. 2-9, 1999.

[3] Alexandru M. Morega, Nicolae Tanase, Mircea Popa, Mihaela Morega, and Jean B. Dumitru, "Numerical simulation of an electromagnetic bending-mode cantilever microactuator", in Proc. of the 8th International Symposium on Advanced Topics in Electrical Engineering (ATEE), Bucharest, ROMANIA, 23-25 May 2013, DOI: 10.1109/ATEE.2013.6563478.

[4] Constantin I. Mocanu, "Electromagnetic Field Theory", 2nd ed., Bucharest, Romania, Ed. D.P., 1982.

[5] Alexandru M. Morega, Nicolae Tanase, Mihaela Morega, Constantin D. Comeaga and Cristinel Ilie, "Bending mode cantilever actuators for microelectromechanical systems", in Proc. of the 9th International Symposium on Advanced Topics in Electrical Engineering (ATEE), Bucharest, ROMANIA, 07-09 May 2015, DOI: 10.1109/ATEE.2015.7133869.

[6] Alexandru M. Morega, Mihaela Morega, Constantin D. Comeaga and Alin Dobre, "Sizing relations for an electromagnetic cantilever actuator", in Proc. of the 9th International Symposium on Advanced Topics in Electrical Engineering (ATEE), Bucharest, ROMANIA, 06-08 October 2016, DOI: 10.1109/ICATE.2016.7754662.

[7] Dumitru Sergiu, Constantin Anghel, Constantin D. Comeaga, Alexandru M. Morega, "Modelling and Simulation of MEMS Electromagnetic Scanner Control", International Conference on Mechanical System and Control, 19-21 May 2017, Sankt Petersburg, Russian Federation. 